\title{
Periodic arrays of pinning centers in thin Vanadium films
}

\author{
V. Metlushko, U. Welp, and G. Crabtree \\ Materials Science Division, Argonne National Laboratory, Argonne, IL 60439 \\ Zhao Zhang and S.R.J. Brueck \\ University of New Mexico, Albuquerque, NM 87131
}

B. Watkins and L.E. DeLong

University of Kentucky, Lexington, $K Y$ 40506-0055

B. Ilic, K. Chung, and P.J. Hesketh

EECS, University of Illinois at Chicago, Chicago, IL 60607

The submitted manuscript has been created
by the University of Chicago as Operator of
Argonne National Laboratory ("Argonne")
under Contract No. W-31-109-ENG-38 with
the U.S. Department of Energy. The U.S.
Government retains for itself, and others act-
ing on its behalf, a paid-up, nonexclusive,
irrevocable worldwide license in said article
to reproduce, prepare derivative works, dis-
tribute copies to the public, and perform pub-
licly and display publicly, by or on behalf of
the Government.

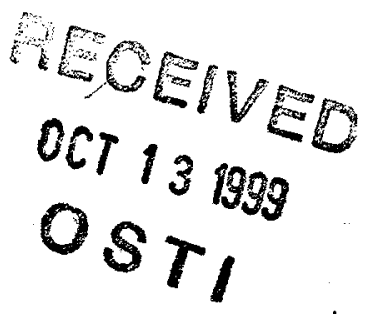

Proceedings of the 43rd Annual International Symposium on Optical Science, Engineering, and Instrumentation (SPIE), July 19-24, 1998, San Diego, CA

This work supported by the U.S. Department of Energy, Basic Energy Sciences-Material

Sciences under contract \#W-31-109-ENG-38 (VM, UW, GC): by DARPA (ZZ, SRJB); and by

U.S. DOE BES-Materials Sciences, Grant DE-FG02-97ER45653 (BW, LED). 


\section{DISCLAIMER}

This report was prepared as an account of work sponsored by an agency of the United States Government. Neither the United States Government nor any agency thereof, nor any of their employees, make any warranty, express or implied, or assumes any legal liability or responsibility for the accuracy, completeness, or usefulness of any information, apparatus, product, or process disclosed, or represents that its use would not infringe privately owned rights. Reference herein to any specific commercial product, process, or service by trade name, trademark, manufacturer, or otherwise does not necessarily constitute or imply its endorsement, recommendation, or favoring by the United States Government or any agency thereof. The views and opinions of authors expressed herein do not necessarily state or reflect those of the United States Government or any agency thereof. 


\section{DISCLAIMER}

Portions of this document may be illegible in electronic image products. Images are produced from the best available original document. 


\title{
Periodic arrays of pinning centers in thin Vanadium films
}

\author{
V.Metlushko, U.Welpa, G.Crabtree ${ }^{a}$ Zhao Zhang ${ }^{b}$, S.R.J.Brueck, B.Watkins', \\ L.E.DeLong, B.Ilic ${ }^{d}$, K.Chung ${ }^{d}$, P.J.Hesketh ${ }^{d}$, \\ ${ }^{2}$ Materials Science Division, Argonne National Laboratory, Argonne, Il 60439-4845 \\ 'University of New Mexico, Albuquerque, NM 87131 \\ University of Kentucky, Lexington, KY 40506-0055 \\ ${ }^{d}$ EECS, University of Illinois at Chicago, 60607
}

\begin{abstract}
Commensurability effects between the superconducting flux line lattice and a square lattice (period $\mathrm{d}=1 \mu \mathrm{m}$ and diameter $\mathrm{D}=0.4 \mu \mathrm{m}$ ) of submicron holes in $1500 \AA$ vanadium films were studied by atomic force microscopy, DC magnetization, AC susceptibility, magnetoresistivity and I-V measurements. Peaks in the magnetization and critical current at matching fields are found to depend nonlinearly upon the value of external AC field or current, as well as the inferred symmetry of the flux line lattice.
\end{abstract}

Keywords: superconductivity, thin films, pinning centers

The value of the critical current in superconducting materials is determined by the balance of Lorenz forces and pinning forces acting on the flux lines. Lorenz forces which are proportional to the current flow tend to drive the flux lines into motion, which dissipates energy and destroys zero resistance (Fig. la). Pinning forces created by isolated defects in the microstructure oppose flux line motion and increase the critical current. Many kinds of artificial pinning centers have been proposed and developed to increase the critical current, ranging from the dispersal of small non-superconducting second phases to creation of defects by proton, neutron or heavy ion irradiation. In all of these methods, the pinning centers are randomly distributed over the superconducting material, causing them to operate well below their maximum efficiency. We overcome this drawback by creating pinning centers in a periodic lattice (see Fig 1b) so that each pin site interacts strongly with only one or a few flux lines. 


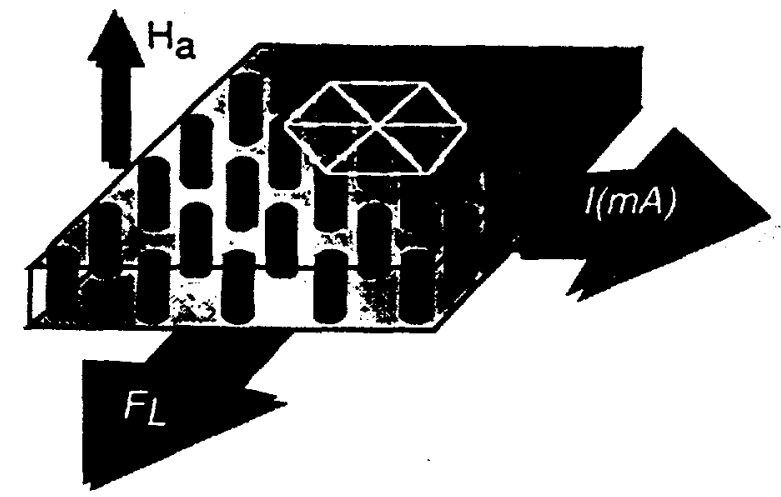

Fig.la. Schematic presentation of vortex lattice in type-II superconductors in the absence of disorder

Fig. 1b. AFM image of a square lattice of holes in vanadium superconducting film

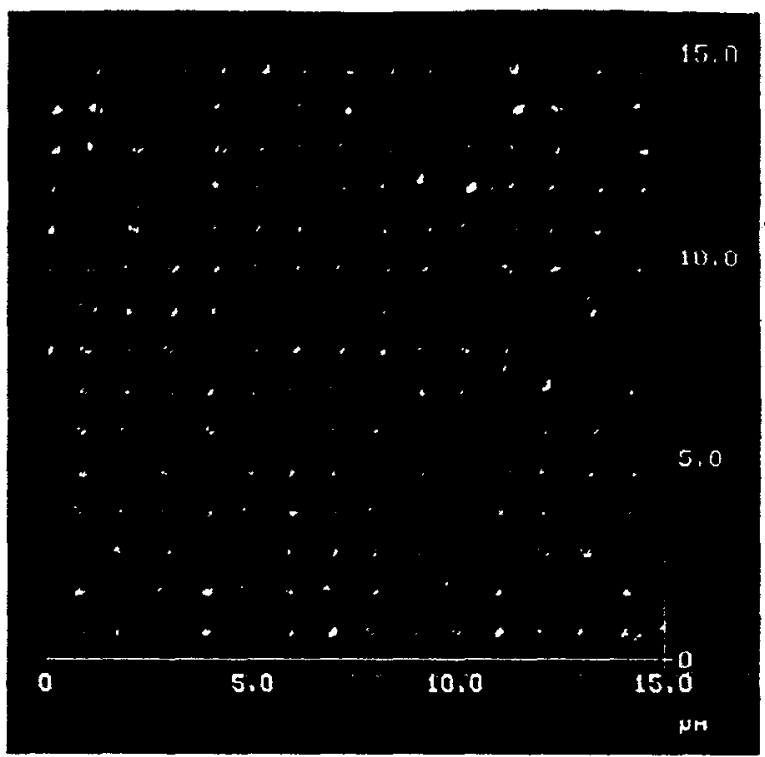

The introduction of periodic arrays of artificial pinning centers into superconducting samples has been shown to give rise to new kinds of vortex behavior ${ }^{1-4}$ that is not observed in the presence of random pinning, e.g., due to material disorder or irradiation damage. Commensurability effects between the pin array (Fig.1b) and the vortex lattice (Fig.la) lead to greatly enhanced pinning and critical currents at a "matching" value of the magnetic induction, $B_{1}=\mu_{0} H_{1}$, for which the intervortex distance, $a_{v}$, equals the period of the pin array, d. At this value of $B$, exactly one vortex resides on each pin site. Any additional vortex generated by increasing the applied field will be accommodated either as a multiple flux quantum per pin site or as an interstitial vortex residing in the superconducting material between the periodic pin sites. For a square array, $\mathrm{H}_{1}=\Phi_{0} / \mathrm{d}^{2}$. The stability of various interstitial vortex arrangements ${ }^{3}$, noise patterns and hysteresis in the IV-characteristics, finite transverse critical currents of the moving vortex system and characteristic narrow band noise spectra have been predicted in recent numerical studies ${ }^{6}$.

Different types of periodic pinning centers that have been studied are micro-holes ${ }^{1.2}$, thickness modulations $s^{4}$ and magnetic dots ${ }^{3}$. Here we investigate the effect of lattices of micto-holes on the vortex dynamics in vanadium films. The characteristic size of micro-holes should be of the same order as the superconducting coherence length $\xi(T)=\xi_{\delta} /\left(1-T / T_{c}\right)^{1 / 2}\left(\approx 100 \mathrm{~nm}\right.$ for vanadium at temperatures close to $\left.T_{c}\right)$ to provide effective FL pinning. This imposes limitations on the fabrication technique used to make such pinning centers, and up to now, only electron beam lithography (EBL) has been employed to perform this task ${ }^{1-4}$. The main drawback to EBL is that it is too slow and expensive to pattern large enough areas necessary for useful sample fabrication. An attractive alternative method for patterning films developed in the present study is based on laser interferometric lithography (IL) ${ }^{7.12}$. 


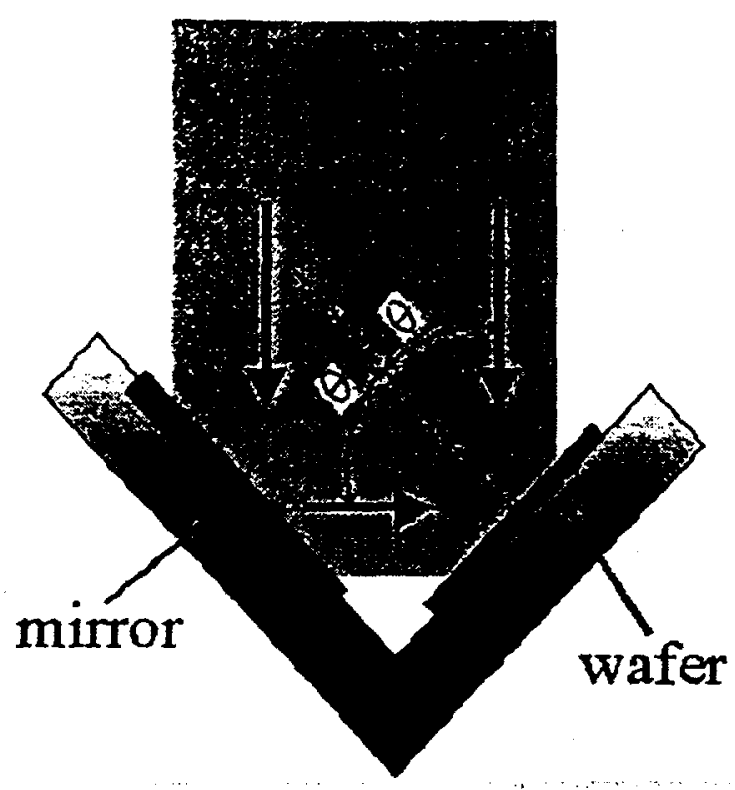

Fig.2. Schematic layout of interferometric lithography

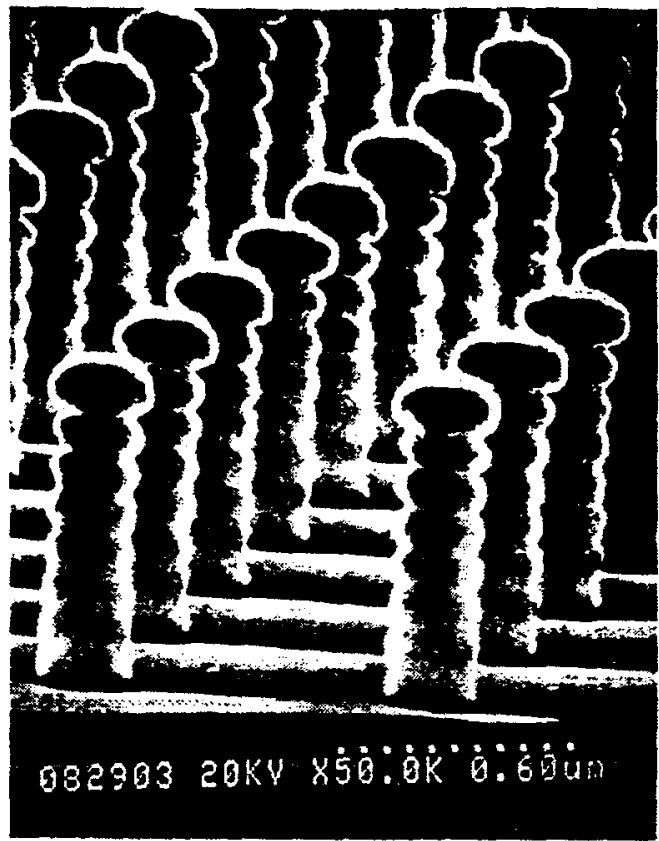

Fig.3. SEM image of square lattice of photoresist posts on $\mathrm{Si} / \mathrm{SiO}_{2}$ substrate after developing

The main principle of $\pi$ is shown in Fig.2. A silicon wafer with a $100-n m$ thick, thermally grown $\mathrm{SiO}_{2}$ insulating layer is spin coated with a $\sim 0.8 \mu \mathrm{m}$ thick novolak-based I-line photoresist (Shipley510). The wafer is exposed to the interference pattern created by two coherent, equal-intensity plane waves derived from a single laser source $\left(\lambda=364 \mathrm{~nm}\right.$ from a $\mathrm{TEM}_{00} \mathrm{Ar}$-ion laser). The plane waves are symmetrically incident about the wafer normal with the separation angle of $2 \theta$. A simple $90^{\circ}$ Fresnel mirror arrangement provides stability for these exposures without requiring an active control loop and also ensures equal intensities. The period is selected by rotating the entire assembly. The resulting aerial image is sinusoidal with a period of $\lambda / 2 \sin \theta$. Two exposures with the wafer rotated by $90^{\circ}$ yield a square array of photoresist dots. A triangular array could be formed by three exposures with $60^{\circ}$ rotations. A post-exposure bake (hotplate, $110^{\circ} \mathrm{C}$ for $60 \mathrm{~s}$ ) is used to strengthen the resist to avoid surface-tension induced collapse on developing these high aspect ratio structures ${ }^{10}$. Non-linearities in the exposure and development processes transform the sinusoidal exposure profile into nearly vertical sidewalls for the final developed photoresist profile (Fig.3). Before development, the samples are soaked in chlorobenzene for $60 \mathrm{sec}$ to retard the development of the pillar tops and create an undercut photoresist structure suitable for the subsequent lift-off process. Using e-beam evaporation a vanadium film is deposited onto the array of photoresist pillars, in this case a $1 \mu \mathrm{m} \times 1 \mu \mathrm{m}$ square array with $0.4 \mu \mathrm{m}$ pillar diameter, Fig.3. After lift-off a vanadium film containing the square array of holes is obtained (Fig.1b and Fig.4). This film is patterned into the final structure using standard optical lithography and wet chemical or reactive ion etching (Fig.4). $2 \times 2 \mathrm{~mm}^{2}$ square samples for magnetization measurements and bridges for transport measurements as shown in Fig. 4 were prepared. Au 
wires were attached using silver epoxy and postbaking. The part of the same substrate without photoresist pillars was used to fabricate a non-perforated reference film. DC-magnetization and AC-susceptibility of the sample were determined using a commercial SQUID magnetometer. The transport characteristics were measured on bridges such as shown in Fig. 4 employing DC and AC currents.
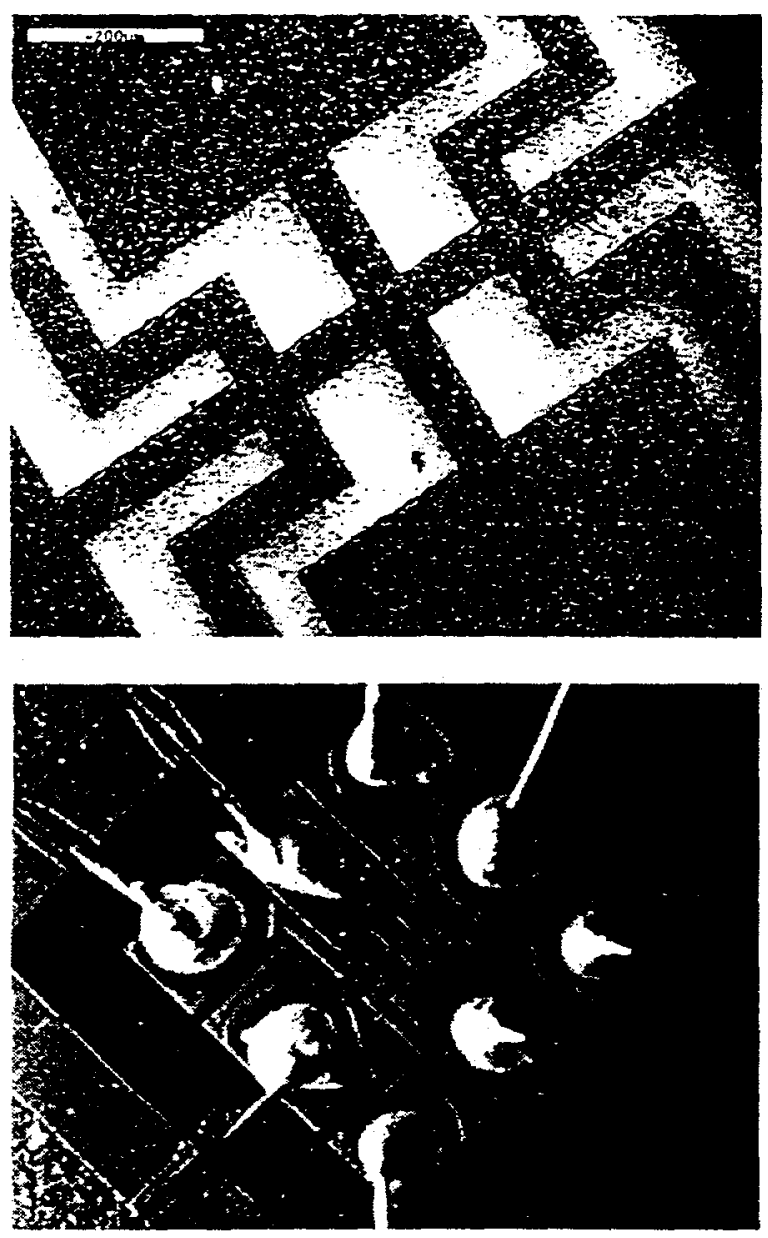

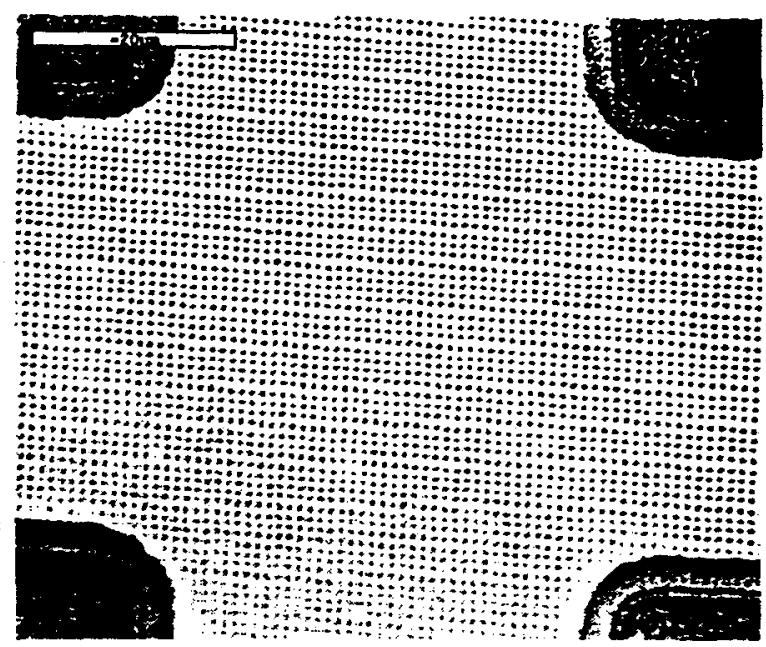

Fig.4a. SEM image of $1500 \AA$ vanadium film with square lattice of holes (period $\mathrm{d}=1 \mu \mathrm{m}$ and diameter $D=0.4 \mu \mathrm{m}$ ) patterned in the bridge for transport measurements

Fig. 4 b. Central part of the vanadium bridge. Rows of holes parallel to the bridge sides are clearly visible

Fig.4c. Optical image of patterned vanadium film with attached Au-contacts

Fig. 5a shows the temperature dependence of the resistivity and susceptibility $\chi=\chi^{\prime}+\mathrm{i} \chi^{\prime \prime}$ in the static field of $1 G$ for a $1500 \AA$ thick vanadium film containing a $1 \times 1 \mu \mathrm{m}^{2}$ square lattice of holes with diameter $D=0.4 \mu \mathrm{m}$ indicating a superconducting transition temperature $T_{c} \approx 4.1 \mathrm{~K}$. This value is lower than $T_{i}=5.43 \mathrm{~K}$ for vanadium single crystal ${ }^{13}$ and close to those reported for thin vanadium films ${ }^{14 \cdot 16}$. The resistivity $\rho$ at $\mathrm{T}>\mathrm{T}_{i}$ is $28 \mu \Omega \mathrm{cm}$ and close to the values reported in the literature ${ }^{17}$. The maxima in $\chi^{\prime \prime}$ at $T=4.02 \mathrm{~K}$ shows that for $T>4.02 \mathrm{~K}$ the $A C$ field with amplitude $h_{0}=0.2 \mathrm{G}$ penetrates to the center of the sample during each cycle. The introduction of the hole array causes a large increase of the magnetic response as shown in a comparison of the susceptibility of an unperforated reference sample and a perforated sample of similar size (Fig. 5bi. In addition, structure in the field dependence of the susceptibility develops which will be discussed in detail below. 


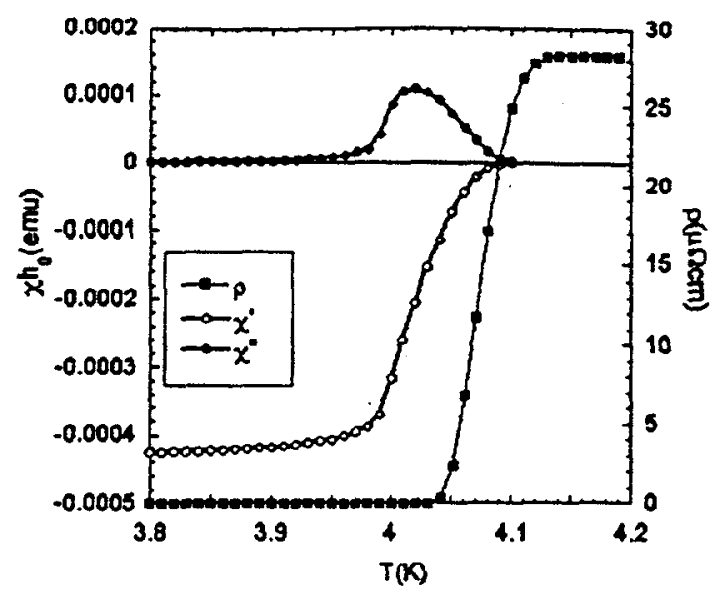

Fig.5a. Temperature dependencies of the susceptibility $\chi=\chi^{\prime}+i \chi^{\prime \prime}$ plotted as $\chi h_{0}$ where $h_{0}=$ $0.02 \mathrm{G}$ is the amplitude of the ac-drive, $\omega=10 \mathrm{~Hz}$ and temperature dependence of the resistivity $\rho$ in a static field $\mathrm{H}=1 \mathrm{G}$ for a vanadium film containing a square lattice of holes

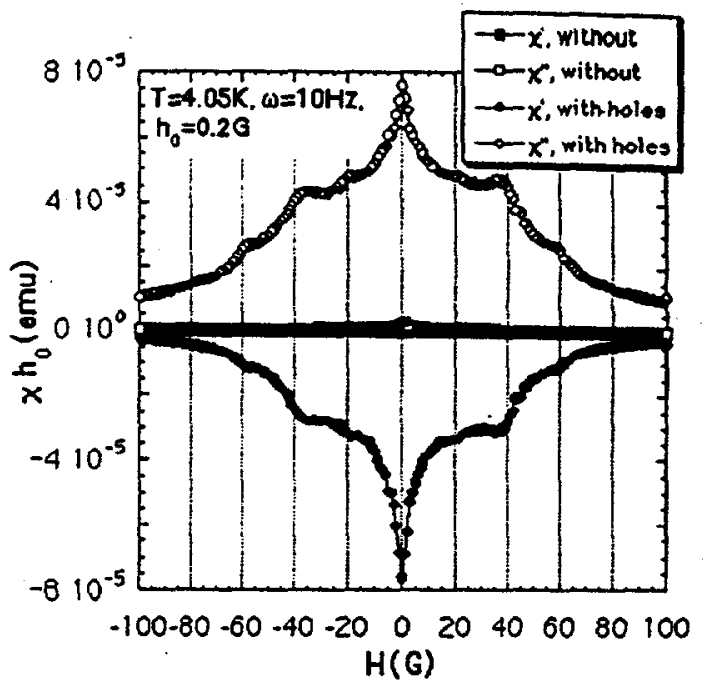

Fig.5b. Field dependenciẹs of susceptibility $\chi h_{0}$ for vanadium film with and without square lattice of holes at $\mathrm{T}=4.05 \mathrm{~K}, \mathrm{~h}_{0}=0.2 \mathrm{G}, \omega=10 \mathrm{~Hz}$

The two components of the complex $A C$ susceptibility $\chi=\chi^{\prime}+i \chi^{\prime \prime}$ reflect the screening current in the sample $\left(\chi^{\prime}\right)$ and losses $\left(\chi^{\prime \prime}\right)$, respectively. The dependencies of the susceptibility on the applied DC field and on $\mathrm{AC}$ drive at $4.06 \mathrm{~K}$ are shown in Figs. $6 \mathrm{a}$ and $6 \mathrm{~b}$. A sequence of sharp minima in $\chi^{\prime}$ at applied fields $H_{n}=n H_{1}$ is observed, where $n= \pm 1, \pm 2$, and \pm 3 , and $H_{1}=20.7 \mathrm{G}$ is the matching field for a $d=1 \mu \mathrm{m}$ square lattice. These minima in $\chi^{\prime}$ are caused by pronounced maxima in the field dependence of the critical current density occurring at the matching fields. This behavior is typically observed in samples containing periodic arrays of pinning sites ${ }^{2.3}{ }^{18}$. For small AC-drive amplitudes $h_{0}$ (Fig.6a), the minima in $\chi^{\prime}$ correspond to minima in $\chi^{\prime \prime}$, where $n= \pm 1 ; \pm 2$ (the case of $n \pm 3$ will be discussed below). This implies that a maximum in the critical current corresponds to a minimum in the AC-losses, as may be expected. However, with increasing drive (Fig.6b) the minima in $\chi^{\prime \prime}$ at $H_{n}=n H_{1}$ transform into maxima. This behavior can be understood by realizing that the AC-losses are determined by the area of MH-hysteresis loops that are traced out in each cycle of the AC-drive ${ }^{19}$. 


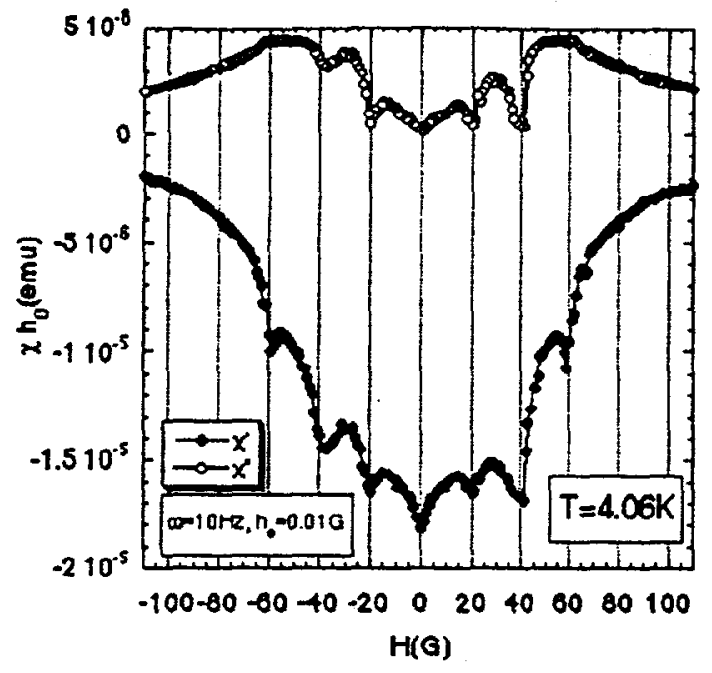

Fig.6a. AC susceptibility $\chi h_{0}$ for vanadium film with square lattice of holes, $A C$ field amplitude $h_{0}=$ $0.01 \mathrm{G}, \omega=10 \mathrm{~Hz}, \mathrm{~T}=4.06 \mathrm{~K}$. In-phase component $\left(\chi^{\prime} h_{0}\right)$ is shown as filled symbols, the out of phase component $\left(\chi^{\prime \prime} h_{0}\right)$ as open circles

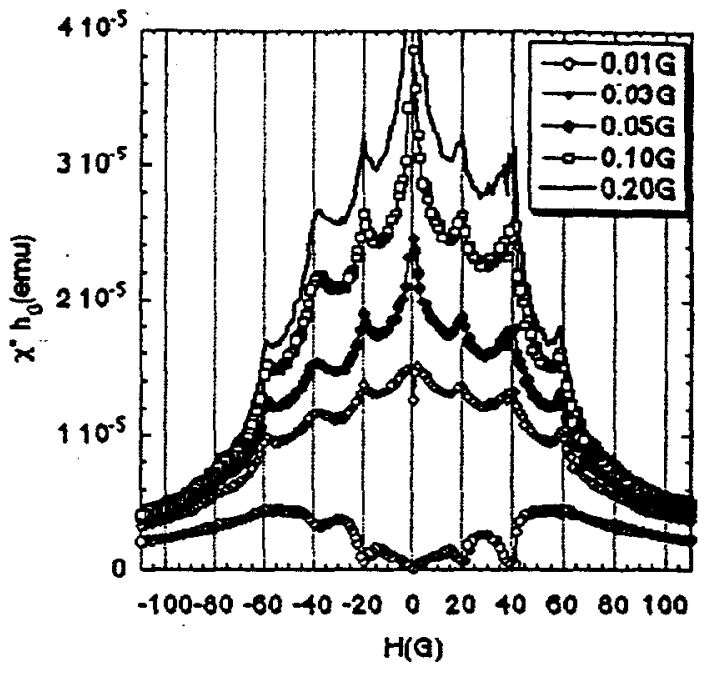

Fig.6b. Field dependence of the out-of-phase component $\left(\chi / h_{0}\right)$ for various ac-drive amplitudes at $\omega=10 \mathrm{~Hz}$ and $\mathrm{T}=4.06 \mathrm{~K}$.

Fig.7 shows schematically the response of weak- and strong-pinning superconductors to a low (a), and to a high drive (b), respectively. At low drives the strong-pinning material effectively screens the AC-field, resulting in linear response and a very narrow hysteresis loop. In contrast, sizable flux penetration occurs in the weak-pinning material, causing hysteretic losses and non-linear response. This situation is observed in Fig.6a by increasing the applied field from, e.g., $\mathrm{H}_{n}=1 \mathrm{H}_{1}$ to $\mathrm{H}_{n}=1.5 \mathrm{H}_{1}$. If the AC-drive is increased to such a level that flux penetration also occurs in the strong-pinning material (Fig.6b), then a larger hysteresis loop accompanied by higher AC-losses occurs, arises due to stronger pinning. This is indeed observed, as shown in Fig. 6b. The onset of non-linearity and enhanced AC-losses in a fixed DC-field of magnitude $\mathrm{H}_{1}$ is apparent for $\mathrm{AC}$-amplitudes $\mathrm{h}_{0}=0.01 \mathrm{G}$, as shown in Fig.8, in agreement with the results in Fig. 6. It can be expected that with increasing $\mathrm{H}=\mathrm{n} \mathrm{H}_{1}$ this onset field decreases, due to the field dependence of $j_{c}$. This suggests that the absence of dips in $\chi^{\prime \prime}$ for $n= \pm 3$ (see Fig. 6a) is caused by a crossover into the strongly non-linear regime at this applied field. 

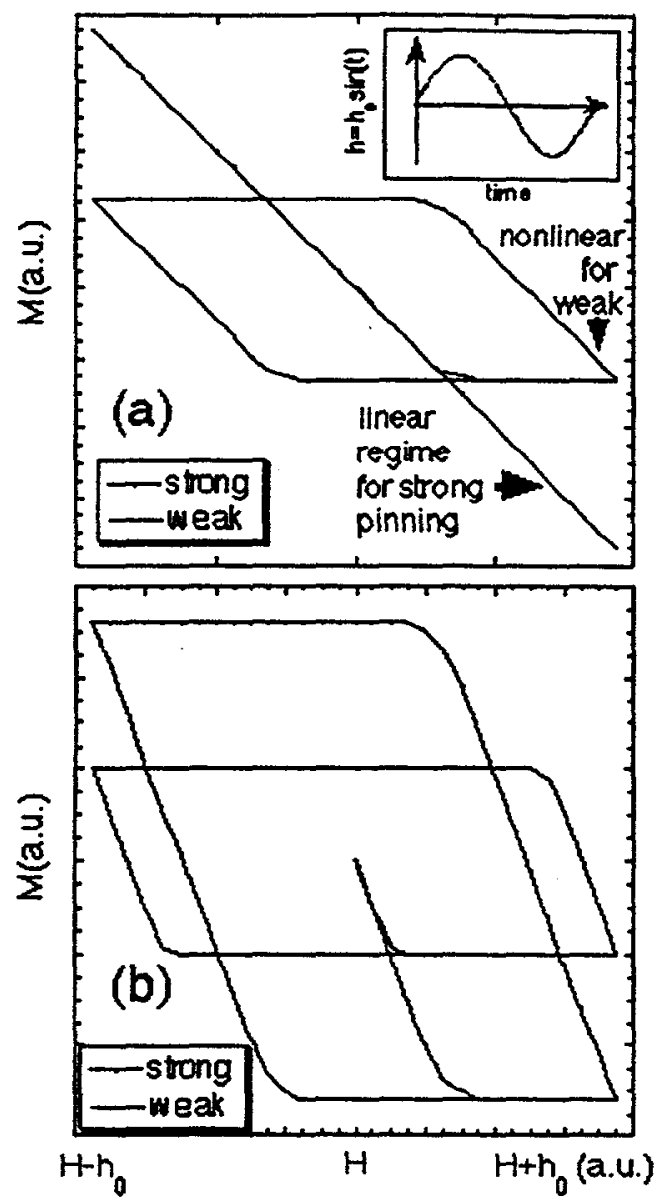

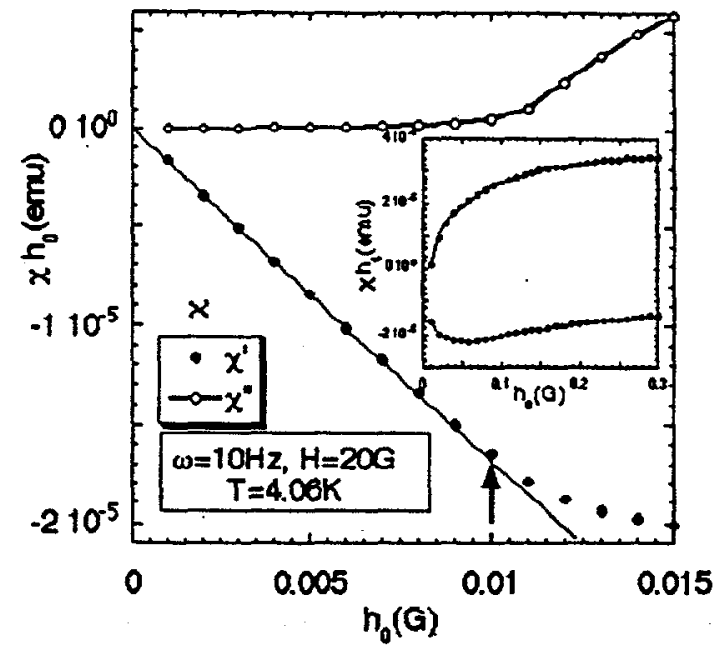

Fig.8. Drive dependence of $\chi \mathrm{h}_{0}$ at the matching field $\mathrm{H}_{1}, \mathrm{~T}=4.06 \mathrm{~K}, \omega=10 \mathrm{~Hz}$. The arrow separates linear regime: $\chi^{\prime} \propto h_{0}, \chi^{\prime \prime}=0$ for $h_{0}<0.01 G$ from the non-linear regime for higher drives. Inset shows $\chi h_{0}$ vs. $h_{0}$ for higher drives

Fig.7. Schematic diagram of the cross-over between linear and non-linear response to a sinusoidal drive field (inset Fig. 6a). a) small ac-drives $h_{0}$ : linear regime for strong pinning and open hysteresis loop (non-linear response) for weaker pining. b) high ac-drives $h_{0}$ : larger area of the hysteresis loop corresponds to stronger pinning.

The magnitudes of the anomalies in $\chi(H)$ at $H_{a}=n H_{1}$ exhibit only a weak logarithmic frequency dependence observed over 5 decades of frequency (see inset in Fig.9). This frequency dependence is characteristic for thermally activated creep. In an AC-experiment the electric field inside the sample is largely determined by the time varying applied field. In this case the normalized flux creep rate can be approximated $^{18}$ as $S \approx \frac{d\left(\ln \chi^{\prime}\right)}{d(\ln \omega)}$, resulting in $S=0.026$ for $\mathrm{H}=\mathrm{H}_{1}$, and $S=0.028$ for $\mathrm{H}=1.5 \mathrm{H}_{1}$. These results agree well with those previously studied in $[\mathrm{Pb} / \mathrm{Ge}]_{\mathrm{n}}$ multilayers ${ }^{2}$ and WGe films ${ }^{18}$ with square lattices of holes. 


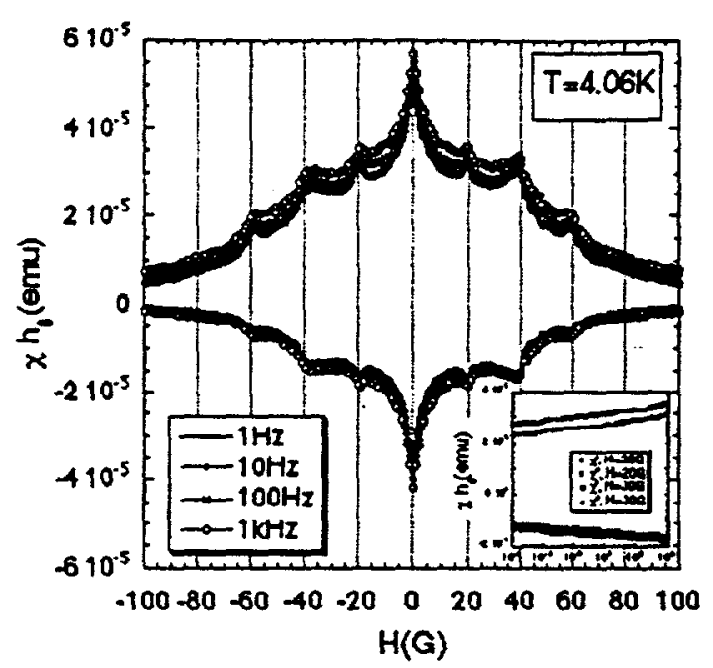

Fig.9. Field dependence of susceptibility $\chi h_{0}$ for a vanadium film with square lattice of holes for different frequencies, $h_{0}=0.2 \mathrm{G}, \mathrm{T}=4.06 \mathrm{~K}$. Inset: $\chi$ vs. $\omega, H=H_{1}, H=3 / 2 H_{1}$.

The prominence of the observed matching fields $\mathrm{H}_{\mathrm{n}}=\mathrm{n}_{1}$ is temperature dependent. In Fig.10, we plot the AC susceptibility $\chi(\mathrm{H})$ for different temperatures. The anomalies in $\chi(\mathrm{H})$ at $\mathrm{H}=\mathrm{H}_{\mathrm{a}}$ become less and less pronounced with decreasing temperature, and at $\mathrm{T}=4.04 \mathrm{~K}$ we observe a well defined anomaly only at $\mathrm{H}=\mathrm{H}_{2}$, and much weaker anomalies at $\mathrm{H}_{1}$ and $\mathrm{H}_{3}$. This result is supported by our DC magnetization (Fig.1 la) and transport measurements (Fig.11b), where the resistivity $\rho(H)$ and the $\mathrm{j}_{c}$ data extracted from $\Gamma V$ curves show a similar behavior. The disappearance of the anomaly at $\mathrm{H}_{1}$ can be explained in the following way.

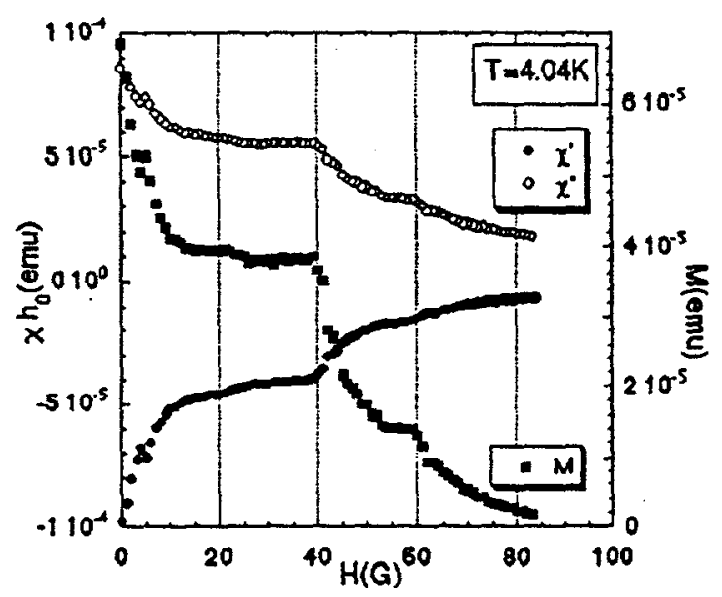

Fig.11a. Field dependencies of the susceptibility $\chi$ $h_{0}$ and $D C$-magnetization $M$ for a vanadium film containing a square lattice of holes at different temperatures, $h_{0}=0.2 \mathrm{G}, \omega=10 \mathrm{~Hz}$.

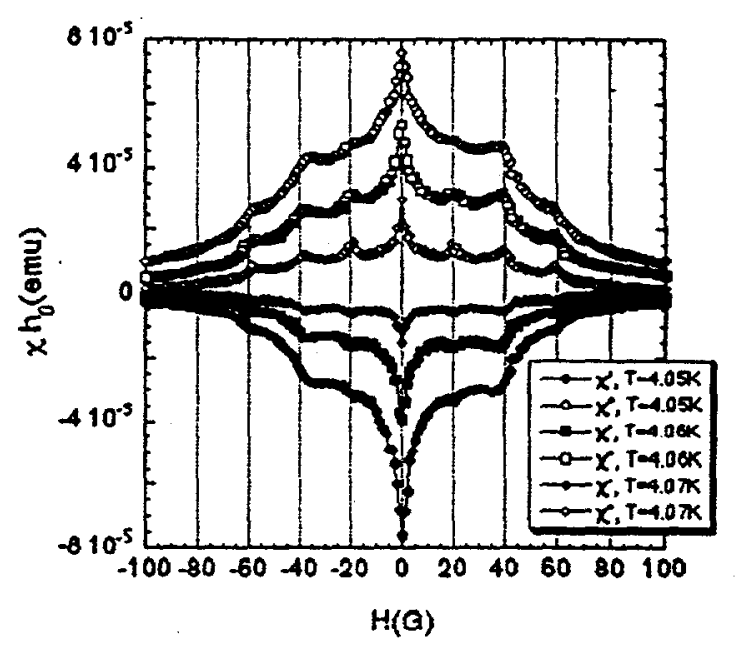

Fig.10. Field dependence of susceptibility $\chi=\chi^{\prime}+$ i $\chi$ " for a vanadium film with square lattice of holes at different temperatures, $h_{0}=0.2 \mathrm{G}, \omega=10 \mathrm{~Hz}$. 
The maximum number of vortices $n_{s}$ which can be trapped in a hole as a multiquantum fluxoid is determined by a simple relation between the hole diameter $D$ and the temperature dependent coherence length $\xi(T): n_{s} \approx D / 4 \xi(T)^{20}$. For our V film, $\xi_{0} \approx 100+440 \AA^{13.16 .17}, T_{c}=4.1 \mathrm{~K}$, and $D=0.4 \mu \mathrm{m}$, yielding $n_{s}<$ 2 in the interval of temperature $\Delta T=T-T_{c}=0.1 \mathrm{~K}$, over which all our measurements were performed. This suggests that we take $n_{s}=1$, and assume that only one FL can occupy a hole and that a second FL will be repelled into the interstices. Based on this, we can identify the anomaly in $\chi$ at $\mathrm{H}=\mathrm{H}_{1}$ as corresponding to a configuration for which each hole has exactly one single- $\Phi_{0}$ fluxoid. $\mathrm{H}=\mathrm{H}_{2}$ corresponds to the situation in which one vortex is in the hole and one vortex resides in the interstices (Fig.12a); and for $\mathrm{H}=\mathrm{H}_{3}$, we have 2 interstitial vortices per unit cell of the hole lattice (Fig.12b). However, the interstitial vortices are not independent from those trapped in the holes. The effective magnetic penetration depth for thin film sample of thickness $t$ is given by $\Lambda(T)=2 \lambda(T)^{2} / t^{21}$. For the temperature range explored here $\Lambda(T)>10 \mu m$ which is much larger than the separation between holes indicating a strong overlap of flux lines and their collective behavior. A consequence of this collective behavior is that at low temperatures the interstitial vortices are effectively "caged" by repulsion from vortices sitting in the holes".

The temperature dependence of the "cage potential" was numerically calculated by Khalfin and Shapiro ${ }^{23}$. They predict that at lower temperatures, a pronounced minimum in free energy $F(x)$ appears at the interstitial site ${ }^{23}$ causing strong interstitial pinning for $\mathrm{H} \approx \mathrm{H}_{2}$. However, for $\mathrm{H}_{2}<\mathrm{H}<\mathrm{H}_{3}$ a second vortex enters the interstitial region giving rise to two possible orientations of the interstitial vortex pair as shown Fig.12b. The activation energy for rotation between these two states is rather weak leading to an unstable vortex arrangement and suppression of the critical current for $\mathrm{H}_{2}<\mathrm{H}<\mathrm{H}_{3}$ (Fig. 10). For $\mathrm{H}_{3}<\mathrm{H}<$ $\mathrm{H}_{4}$ interstitial vortices form a slightly distorted triangular lattice around holes. Recently, these vortex configurations have been directly imaged using e-beam holography $y^{24}$. This configuration is relatively stable and changes in $\mathrm{L}_{c}(\mathrm{H})$ and $\rho(\mathrm{H})$ at $\mathrm{H}=\mathrm{H}_{3}$ are not as pronounced as for $\mathrm{H}=\mathrm{H}_{2}$.
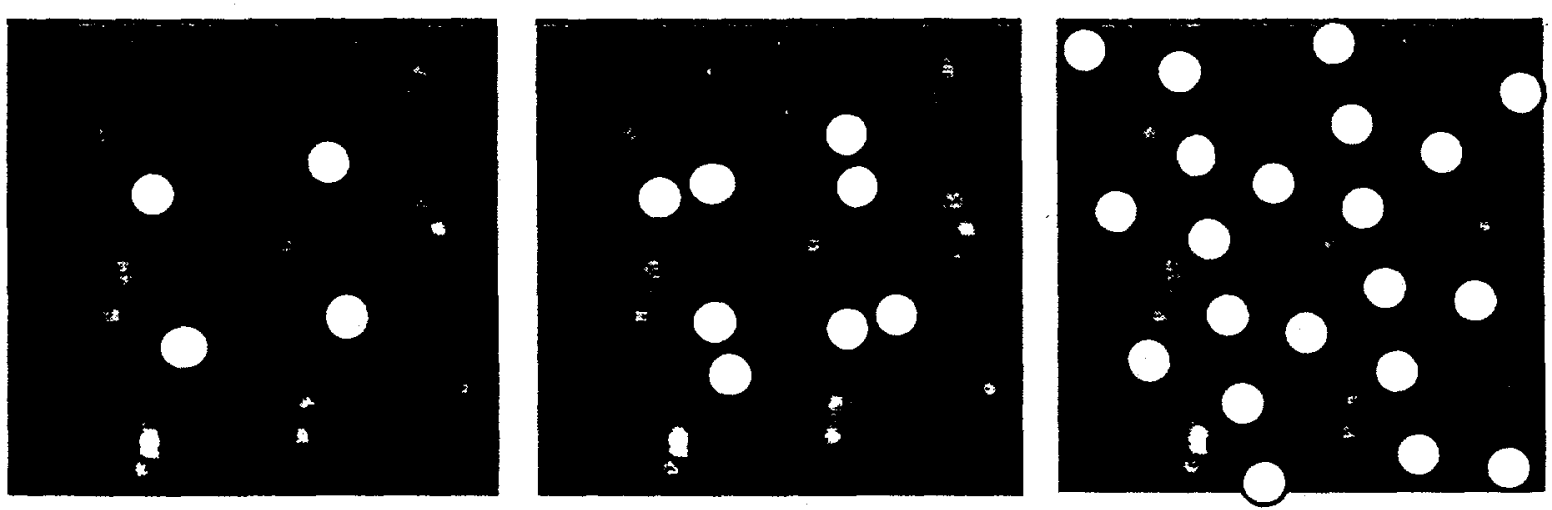

Fig. 12. Vortex configurations for: (a) $\mathrm{H}_{1}>\mathrm{H} \geq \mathrm{H}_{2}$; (b) $\mathrm{H}_{2}>\mathrm{H}>\mathrm{H}_{3}$; (c) $\mathrm{H}_{3}>\mathrm{H} \geq \mathrm{H}_{4}$. Only "interstitial" vortices (open circles) are shown superimposed on the AFM image of the sample. 
In conclusion, we present a novel fabrication technique for the production of superconducting thin films containing a periodic array of pinning sites in the form of sub-micron holes. This technique is based on laser interferometric lithography. It allows for the fast production of large area samples without the need of joining individually written blocks as is necessary in electron beam lithography. We apply this technique for the fabrication of vanadium thin films containing a square lattice of holes with period of $1 \mu \mathrm{m}$ and diameter of $0.4 \mu \mathrm{m}$. Sharp anomalies in the ac-susceptibility, magnetization and magneto-resistivity are observed at magnetic fields that correspond to the matching of the vortex lattice to the hole lattice. With increasing ac-drive a characteristic change of sharp minima in the field dependence of $\chi$ " (that is, the dissipation) to pronounced maxima is found. This transformation is explained as the cross-over from linear to non-linear vortex response. The magnitude of the anomalies in $\chi$ at the matching fields reflects the difference in stability of various vortex configurations. The sharp decrease in susceptibility and transport critical current for $\mathrm{H}>\mathrm{H}_{2}$ confirms that vortex configurations with two interstitial vortices are less stable than configurations with one or three interstitial vortices.

\section{ACKNOWLEDGMENTS}

This work was supported by the U.S. DOE, BES-Materials Sciences, under contract W-31-109-ENG-38 (V.M., U.W., G.C.), by DARPA (Z.Z., S.R.J.B.), by U.S. DOE BES-Materials Sciences, Grant DEFG02-97ER45653 (B.W., L.E.D)

\section{REFERENCES:}

1. A.T.Fiory, A.F.Hebard, and S.Somekh, Appl. Phys. Lett., 32, 73 (1978).

2. V.V.Metlushko, M.Baert, R.Jonckheere, V.V.Moshchalkov, and Y.Bruynseraede, Solid State Commun. 91, 331 (1994); M.Baert, V.V.Metlushko, R.Jonckheere, V.V.Moshchalkov, and Y.Bruynseraede, Phys. Rev. Lett., 74, 3269 (1995); V.V.Moshchalkov, M.Baert, V.V.Metlushko, E.Rosseel, M.J.Van Bael, K.Temst, R.Jonckheere, and Y. Bruynseraede, Phys. Rev. B, 54, 7385 (1996); V.V.Moshchalkov, M.Baert, V.V.Metlushko, E.Rosseel, M.J.Van Bael, K.Temst, Y. Bruynseraede, and R.Jonckheere, Phys. Rev. B, 57, 3615 (1998).

3. J. I. Martin, M. Velez, J. Nogues, and I. K. Schuller, Phys. Rev. Lett. 79, 1929 (1997); D. J. Morgan, and J. B. Ketterson, Phys. Rev. Lett. 80, 3614 (1998).

4. A.Bezryadin and B.Pannetier, J. Low Temp. Phys. 102, 73 (1996); A.Bezryadin, Yu.N.Ovchinnikov and B.Pannetier, Phys. Rev. B. 53, 8553 (1996).

5. C.Reichhardt, C.J.Olson, F.Nori, submitted to Phys. Rev. B.

6. C.Reichhardt, J.Groth, C.J.Olson, S.B.Field, F.Nori, Phys. Rev. B 54, 16108 (1996)

7. H.Anderson, H.I.Smith, and M.L.Schattenberg, Appl. Phys. Lett. 43, 874 (1983). 
8. S.H.Zaidi, and S.R.J.Brueck, J. Vac. Sci. Technol. B 11, 658 (1993); S.H.Zaidi, A.-S.Chu, and S.R.J.Brueck, J. Appl. Phys. 80, 6997 (1996); X.Chen, S.H.Zaidi, S.R.J.Brueck, and D.J.Devine, J. Vac. Sci. Technol. B 14, 3339 (1996).

9. T.A.Savas, S.N.Shah, M.L.Schattenburg, J.M.Carter, and H.I.Smith, J. Vac. Sci. Technol. B 13, 2732 (1996); A.Fernandez, J.Y.Decker, S.M.Herman, D.W.Phillion, D.W.Sweeney, and M.D.Perry, J. Vac. Sci. Technol. B 15, 2439 (1997).

10. X. Chen, Z. Zhang, S. R. J. Brueck, R. A. Carpio and J. S. Petersen, SPIE Microlithography - 97, Emerging Lithographic Technologies 3048, 309-318 (1997).

11. C.O.Bozler, C.T.Harris, S.Rabe, D.D.Rathman, M.A.Hollis, H.I.Smith, J. Vac. Sci. Technol. B 12, 629 (1994).

12. A.Fernandez, H.T.Nguyen, J.A.Britten, R.D.Boyd, M.D.Perry, D.R.Kania, and A.M.Hawryluk, J. Vac. Sci. Technol. B 15, 729 (19970).

13. S.T.Sekula, R.H.Kernohan, Phys. Rev. B 5, 904 (1972).

14. P.Koorevaar et al., Phys. Rev. B 47, 934 (1993).

15. Y.Kuwasawa, E.Touma, T.Nojima, and S.Nakano, Physica B 194-196, 2423 (1994).

16. A.A.Teplov, M.N.Micheeva, Sov. J. Low. Temp. Phys. 7, 149 (1981).

17. K.Kanoda, H.Mazaki, T.Yamada, N.Hosoito, and T.Shinjo, Phys. Rev. B 33, 2052 (1986); K.Kanoda, H.Mazaki, N.Hosoito, T.Shinjo, Phys. Rev. B 35, 6736 (1987).

18. V.V.Metlushko, L.E.DeLong, M.Baert, E.Rosseel, M.J.Van Bael, K.Temst, V.V.Moshchalkov, and Y.Bruynseraede, Europhysics Letters 41, 333 (1998),

19. J.Clem, Magnetic susceptibility of superconductors and other spin systems (Plenum Press, New York, 1991, Ed. R.A.Hein).

20. G.S.Mkrtchyan and V.V.Shmidt, Sov. Phys. JETP 34, 195 (1972).

21. R.D. Parks, Superconductivity (Marcel Dekker, Inc., New York, 1969).

22. L.Radzihovsky, Phys. Rev. Lett., 74, 4923 (1995).

23. I.B. Khalfin and B.A.Shapiro, Physica C 207, 359 (1993).

24. K.Harada, O.Kamimura, H.Kasai, T.Matsuda, A.Tonomura, and V.V.Moshchalkov, Scince 274, 1167 (1996). 